\title{
Ischial Tuberosity Tuberculosis : An Unusual Location and Presented as Chronic Gluteal Abscess
}

\author{
H Krishnan, MS Ortho, TR Yoon*, MD, KS Park*, MD, YJ Cho*, MD \\ Orthopaedic Department, Queen Elizabeth Hospital. Kota Kinabalu, Malaysia \\ ${ }^{*}$ Center for Joint Disease, Chonnam National University Hwasun Hospital, Jeonnam, Korea
}

\begin{abstract}
This case report describes an unusual case of extrapulmonary tuberculosis of the ischial tuberosity presenting with chronic gluteal pain of 4 years duration. The patient presented when the pain became intolerable; at that time, the ischial tuberosity was debrided and curetted while incision and drainage were used to treat the gluteal abscess. Antituberculosis chemotherapy was administered (for a period of one year) following histopathological confirmation of tuberculosis. At four years postoperatively, the patient has no pain and is symptom free. Furthermore, radiographs shows that the right ischial tuberosity osteomyelitis has healed. Prompt diagnosis and treatment resulted in a good clinical outcome in this patient.
\end{abstract}

Key Words:

Gluteal Abscess, Ischial Tuberosity Tuberculosis, Anti Tuberculosis Chemotherapy

\section{INTRODUCTION}

Although tuberculosis (TB) is a rare infection in the developed world, in the developing world it is still prevalent. Musculoskeletal tuberculosis constitutes $1-5 \%$ of all TB cases $^{1}$; further, in the past few years there has been an increased incidence of osteoarticular tuberculosis in association with AIDS in developing countries. This increased incidence is due to many factors such as Human Immunodeficiency Virus infection, immigration from endemic areas, alcoholism, chronic kidney disorders, immunosuppressive therapy, homelessness, crowded living conditions, drug addiction, intra-articular steroid injection, systemic illness and poor access to medical care.

The present case describes an unusual location of tuberculosis involving the ischial tuberosity that presented with a chronic gluteal abscess. However, the patient did not have any of the risk factors mentioned in the above paragraph.

\section{CASE REPORT}

A 32-year-old woman presented at our outpatient department with right buttock pain. She has experienced a dull right buttock pain for 4 years, but the pain had increased in intensity and affected the right posterior thigh and gluteal area with associated swelling over the previous 2 months. She decided to seek help because the pain had worsened while sitting, especially over the right ischial tuberosity. The patient had no sciatica-type pain, but did experience pain over the posterior right thigh while walking. Over the past 4 years, she had been taking oral analgesics to control the pain. She denied any previous history of contact with tuberculosis or direct inoculation (intramuscular injection) and had no relevant family history. Furthermore, she experienced no weight loss or decrease in appetite and had no previous history of surgery.

A physical examination conducted at presentation revealed a swelling of $\sim 10 \times 5 \mathrm{~cm}$ running from the right buttock to the proximal right posterior thigh with a poorly defined margin that was non-inflamed, had no sinus, and was mildly tender on deep palpation. Range of motion of the right hip was reduced due to pain (flexion $0-70^{\circ}$, external rotation $0-40^{\circ}$, internal rotation $0-20^{\circ}$, abduction $0-30^{\circ}$, and adduction 0 $\left.10^{\circ}\right)$. No inguinal lymphadenopathy was detected.

A plain radiograph revealed an osteolytic lesion over the right ischial tuberosity and soft tissue swelling around the proximal right femur and hip with areas of calcification (Figure 1). Her erythrocyte sedimentation rate (ESR) was 68 $\mathrm{mm} /$ hour, and her C-reactive protein (CRP) level was 2.4 $\mathrm{mg} / \mathrm{dl}$ (both elevated), but her total white blood cell count was normal at $6100 / \mathrm{mm} 3$. Serology for HIV was negative. Next, the tuberculin test was negative, acidfast bacillus in sputum and urine was negative, and the chest radiograph was clear. However, CT (Figure 2) and MR (Figure 3) imaging showed a multiseptate, multilobular, rim-enhanced mass lesion of $\sim 12 \times 7 \times 13 \mathrm{~cm}$ in the right buttock, with soft tissue changes surrounding the lesion and erosive changes in the right ischial tuberosity. 


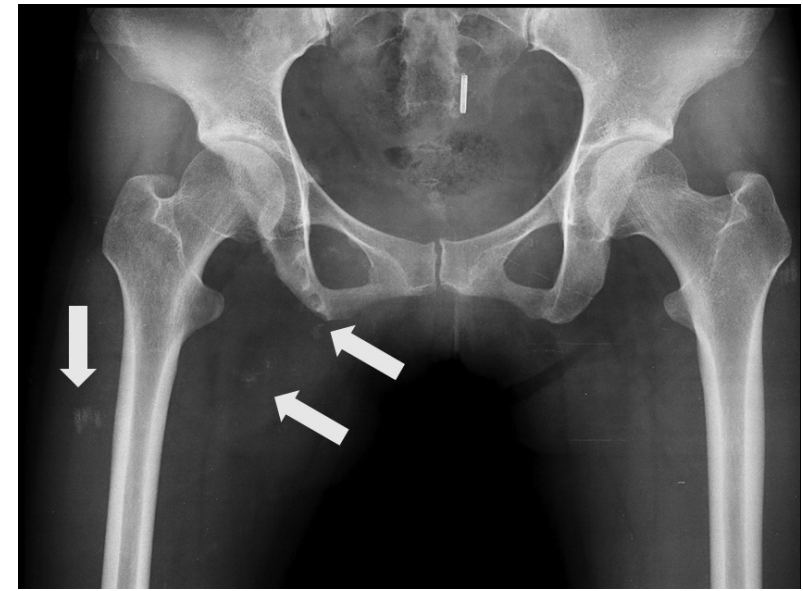

Fig. 1: Osteolytic lesion of the right ischial tuberosity associated with soft tissue swelling and areas of calcification around the proximal right femur.

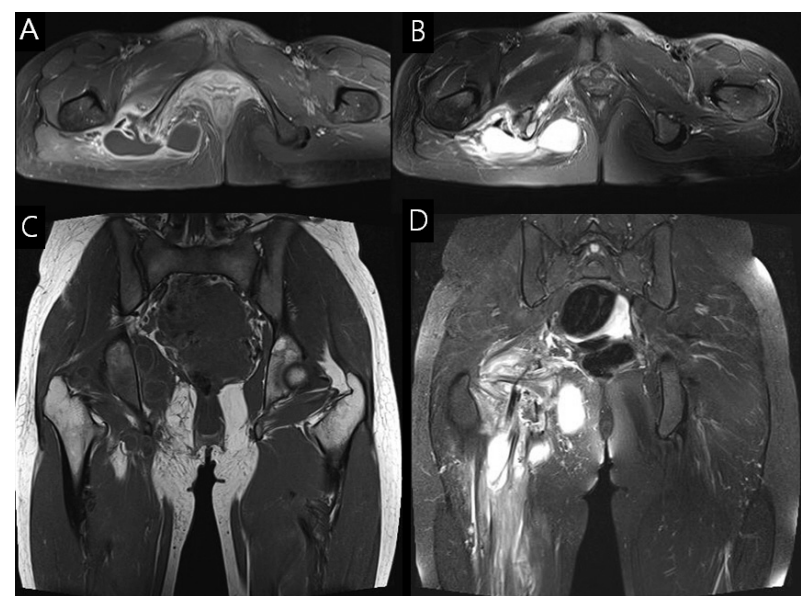

Fig. 3: MRI showing fluid collection and multiloculate and multiseptate involvement (A-D) of right gluteal muscles.

Based on history, clinical exams, blood parameters, and radiological images, the working diagnosis was osteomyelitis of right ischial tuberosity and a chronic gluteal abscess with a high suspicion of tuberculosis. Elective surgery of incision and drainage (gluteal abscess) and curettage (ischial tuberosity osteomyelitis) was performed under general anaesthesia. Intraoperatively, whitish calcified material and about $5 \mathrm{mls}$ of a whitish fluid was seen deep in the gluteus maximus. Accordingly, the ischial tuberosity was curetted and debrided to remove the calcified material and sequestrum. Samples were sent for histopathological examination and culture, which revealed epithelioid cells and Langhan's giant cells consistent with tuberculosis, but negative cultures. She was started on a 12-month course of a four-drug antituberculosis chemotherapy (isoniazid, $5 \mathrm{mg} / \mathrm{kg}$; rifampicin, $10 \mathrm{mg} / \mathrm{kg}$; ethambutol, $15 \mathrm{mg} / \mathrm{kg}$; and, pyrazinamide, $20 \mathrm{mg} / \mathrm{kg}$.

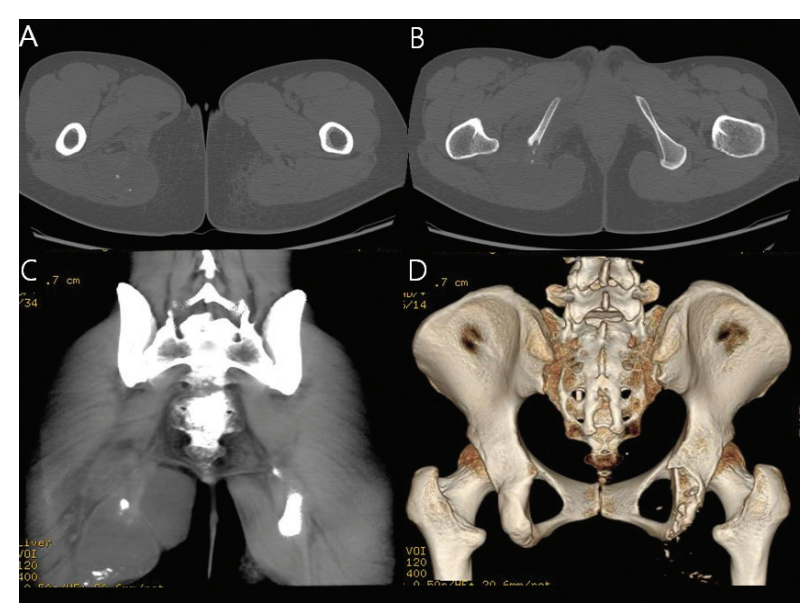

Fig. 2: CT scan showing areas of calcification $(A, C)$ in the right thigh and destruction of the right ischial tuberosity (B,D).

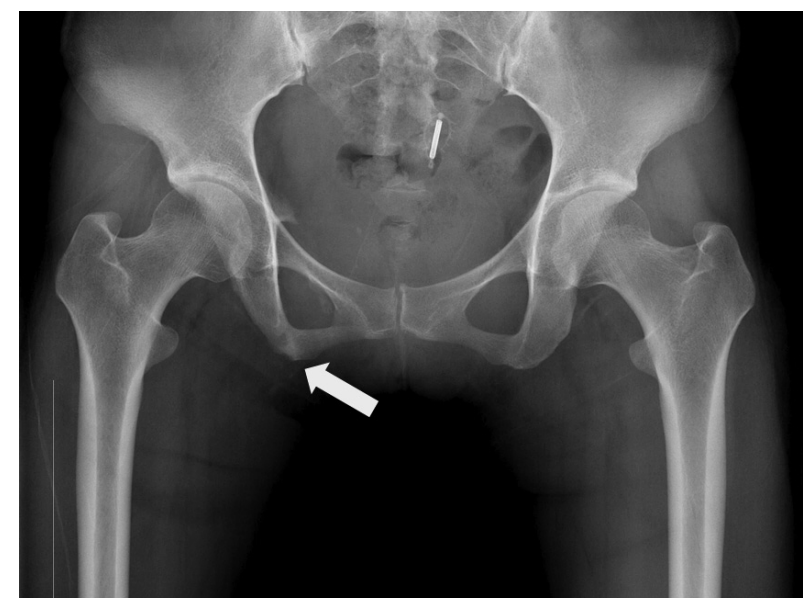

Fig. 4: Radiograph taken at last follow up (4 years postoperatively) showing the healed right ischial tuberosity lesion and the absence of areas of calcification around the right proximal femur.

At her last follow up, 4 years postoperatively, the patient was doing well, had no pain while walking or sitting, and had returned to her normal activities. Clinically, range of right hip motion was full and there was no evidence of swelling of the posterior right thigh or buttock. Plain radiograph revealed consolidation of the osteolytic lesion of the right ischial tuberosity (Figure 4), and laboratory parameters (ESR, CRP) were also normalized.

\section{DISCUSSION}

Tuberculosis involving the ischial tuberosity is rare. Between the years 1901-1935, only seven cases were reported (all in French) in the literature and in 1936 Kaplan described the first case in English literature ${ }^{2}$. This was followed by Magnusson (1938) in an article in which he estimated the incidence of ischial tuberosity tuberculosis to be $0-2$ per cent of all cases of bone tuberculosis ${ }^{2}$. 
A review of patients with skeletal tuberculosis by Silva ${ }^{3}$ (1980) showed only one case of ischial tuberosity tuberculosis out of 219 cases of tuberculosis involving bones and joints. More recently in 2008, Bhattacharyya ${ }^{4}$ reported three cases of ischial tuberosity tuberculosis and all cases were treated by thorough curettage and antituberculosis treatment (with good clinical outcome).

In this present case the gluteal abscess was secondary to tuberculous lesion of the ischial tuberosity. When the patient came to the OPD, the anteroposterior pelvis radiograph showed an osteolytic lesion over the right ischial tuberosity (with gluteal pain and swelling). She denied any history of tuberculosis or direct inoculation. Bhattacharyya ${ }^{4}$ had described that ischial tuberosity tuberculosis usually presents as gluteal pain and swelling with or without a discharging sinus. Derkash ${ }^{5}$ reported that skeletal muscle tuberculosis abscesses are rarely described in isolation and that tuberculosis can involve skeletal muscle by extension from bone, the synovial linings of joints, or tendon sheaths, and is generally contracted by direct inoculation and only rarely via a hematogenous route. In 1997, Hornbuckle ${ }^{6}$ reported a case of mycobacterium tuberculosis presenting as a chronic gluteal abscess. In that case, the diagnosis of a tuberculosis abscess was delayed and as a result, the gluteal muscle abscess developed communicating fistula tracts to the rectum and skin.

Tuberculosis must be ruled out when one is presented with a chronic pain and swelling of any kind. Furthermore, in cases with a high index of suspicion for tuberculosis, other sites must be investigated to enable prompt treatment and prevent complications as described by Hornbuckle ${ }^{6}$. In the present case, the patient presented late with a chronic pain of 4 years and swelling of 2 months duration; the late presentation allowed development of osteomyelitis of the right ischial tuberosity with a chronic gluteal abscess. Tuberculosis was diagnosed promptly, confirmed by histopathological examination, and treatment was initiated. At the last follow up, the patient was clinically well and radiograph showed a healed ischial tuberosity lesion.

In summary, prompt treatment in this case was followed by a good clinical outcome; even in a rare, unusual location of ischial tuberosity tuberculosis with a chronic gluteal abscess. 


\section{REFERENCES}

1. Weir MR, Thornton GF. Extrapulmonary tuberculosis. Experience of a community hospital and review of the literature. $A m J$ Med. 1985; 79: 467-78.

2. Tupman GS. A case of tuberculosis of the ischium. J Bone Joint Surg Br 1953; 35: 590-2.

3. Silva JF. A review of patients with skeletal tuberculosis treated at the university hospital, Kuala Lumpur. International Orthop, 1980; 4: 79-81.

4. Bhattacharyya AN. Tuberculosis of the ischium: A report of three cases. ANZ J Surg 2008; 42: 389-91.

5. Derkash RS, Makley JT. Isolated tuberculosis of triceps muscle- Case Report. J Bone Joint Surg Am 1979; 61: 948.

6. Hornbuckle K, Bonomo Robert A, Armitagee Keith B. Mycobacterium tuberculosis presenting as a chronic gluteal abscess. Infectious Diseases in Clinical Practice. 1997; 6: 413-5. 